Original Research

\title{
Preprocedural features of patients under antihypertensive drugs may help identify responders to renal denervation: a hypothesis-generating study
}

\author{
Shu-I Lin ${ }^{1,2}$, Chun-Che Huang ${ }^{3}$, Shih-Hsien Sung ${ }^{4}$, Lawrence Yu-Min Liu ${ }^{5}$, Po-Lin Lin ${ }^{5}$, \\ Wei-Ren Lan ${ }^{1,2}$, Chuan-Lei Chao ${ }^{6}$, Wei-Ru Chiou ${ }^{2,6}$, Cheng-Ting Tsai ${ }^{1,2,7}$, Yih-Jer $\mathrm{Wu}^{1,2}$, \\ Tzung-Dau Wang ${ }^{8}$, Ying-Hsiang Lee ${ }^{1,2,7, *}$ \\ ${ }^{1}$ Cardiovascular Center, MacKay Memorial Hospital, 104217 Taipei, Taiwan \\ ${ }^{2}$ Department of Medicine, Mackay Medical College, 252 New Taipei, Taiwan \\ ${ }^{3}$ Department of Healthcare Administration, College of Medicine, I-Shou University, 84001 Kaohsiung, Taiwan \\ ${ }^{4}$ Division of Cardiology, Department of Internal Medicine, Taipei Veterans General Hospital, 112 Taipei, Taiwan \\ ${ }^{5}$ Department of Cardiology, Hsinchu MacKay Memorial Hospital, 300 Hsinchu, Taiwan \\ ${ }^{6}$ Department of Cardiology, Taitung MacKay Memorial Hospital, 950 Taitung, Taiwan \\ ${ }^{7}$ Department of Artificial intelligence and Medical Application, MacKay Junior College of Medicine, Nursing, and Management, 11260 Taipei, Taiwan \\ ${ }^{8}$ Department of Internal Medicine, National Taiwan University Hospital, 100 Taipei, Taiwan \\ *Correspondence: speakerlee@gmail.com (Ying-Hsiang Lee) \\ Academic Editors: Stefano Omboni, Brian Tomlinson and Takatoshi Kasai \\ Submitted: 1 December 2021 Revised: 12 January 2022 Accepted: 19 January 2022 Published: 16 February 2022
}

\begin{abstract}
Background: Renal denervation (RDN) is effective to lower systolic blood pressure (SBP) in essential hypertension. However, patient selection under medications remains an important unmet clinical need. Methods: This multicenter study aimed at observing whether preprocedural features associated with increased renin-angiotensin-aldosterone activity influence RDN response. This study enrolled the patients who underwent RDN for uncontrolled hypertension. Medical records were reviewd and patients were divided into 2 groups depending by meeting any of the following conditions prior to RDN: (1) $>10 \mathrm{mmHg}$ of office SBP reduction after aldosterone inhibition, (2) aldosterone-renin ratio $>30$ or (3) slow flow on the renal angiogram. RDN responders were defined by a reduction in 24-hour mean $\geq 6 \mathrm{mmHg}$ or by $\geq 1$ class of antihypertensive drug withdraw. Results: A total of 46 patients were enrolled, of which $27(59 \%)$ were in control group A and 19 (41\%) in group B. The baseline age, gender, office and 24-hour SBP (mean $140.0 \pm 12.8 \mathrm{mmHg}$ vs. $144.0 \pm$ $16.5 \mathrm{mmHg}, p=0.577)$ were comparable, while the number of prescribed drug classes was fewer in group A $(4.0 \pm 1.3 \mathrm{vs} .4 .9 \pm 0.9, p$ $=0.014)$. The proportion patients with prescribed aldosterone antagonist or high aldosterone-renin ratios were higher in group B. At 12 months post RDN, the results were significantly better in group B in terms of mean change in office SBP $(12.4 \pm 23.5 \mathrm{mmHg} v \mathrm{vs} .29 .9$ $\pm 25.5 \mathrm{mmHg}, p=0.046)$ and the proportion of $\mathrm{RDN}$ responders $(51.9 \%$ vs. $89.5 \%, p<0.001)$. Conclusion: RDN was more effective in patients with any of 3 clinical indices.
\end{abstract}

Keywords: aldosteronism; hypertension; patient selection; renal denervation

\section{Introduction}

Several prospective randomized sham-controlled trials have demonstrated that percutaneous renal denervation (RDN) lowers office and 24-hour blood pressure (BP) in patients with uncontrolled hypertension in both the presence and absence of antihypertensive drug therapy [1-3]. This procedure provides an opportunity to achieve BP goals with lower tablet burden [4]. Current clinical interest fucusses on further increasing the RDN response rate. However, the latest evidence focusing on heart rate and renin-angiotensinaldosterone system (RAAS) were all derived from a drugfree population [5-7]. Thus, distinguishing those hypertensive patients on medication who are best suited to the renal denervation procedure remains an unmet need.

A post-hoc analysis of the randomized shamcontrolled Symplicity HTN-3 trial indicated that the use of an aldosterone antagonist at baseline was an independent predictor of the blood pressure response to $\mathrm{RDN}[8,9]$. However, patients with primary aldosteronism were excluded from the Symplicity HTN-3 trial. It is, therefore, possible that $\mathrm{RDN}$ is more effective in patients who tend to continue using an aldosterone antagonist for the underlying overactivity of RAAS [10]. In a pilot proof of concept study, several preprocedural characteristics featuring overactive RAAS on medical treatment were associated with RDN response [11]. The present analysis was aimed at increasing the sample size to compare blood pressure response among RDN recipients with vs. without the three features of overactive RAAS in a multi-center database. 


\section{Methods}

\subsection{Patient population and study design}

We reviewed medical records of consecutive patients who received catheter-based radiofrequency RDN (Symplicity Flex ${ }^{\mathrm{TM}}$ or Spyral ${ }^{\mathrm{TM}}$, Medtronic, Minneapolis, MN, USA) for uncontrolled hypertension in three medical centers between September 2013 and August 2019 under approval of Institutional Review Boards, MacKay Memorial Hospital (IRB No. 20MMHIS087e), which waived the requirement for informed consent in this retrospective study. As of August 2019, patients meeting inclusion criteria were consecutively identified by the same inclusion and exclusion criteria from three hospitals, including the first 18 patients in the prior single-center pilot study [11]. Patient records were de-identified to preserve patient privacy and the study was performed in accordance with the guidelines of ICH-GCP (International Conference on Harmonisation of Technical Requirements for Registration of Pharmaceuticals for Human Use-Good clinical practice). Patients had no previous diagnosis of secondary hypertension, such as primary aldosteronism, although some had a reported history of obstructive sleep apnea and chronic kidney disease (CKD, defined as eGFR [estimated glomerular filtration rate] $\left.<60 \mathrm{~mL} / \mathrm{min} / 1.73 \mathrm{~m}^{2}\right)$. Diabetes was usually diagnosed as HbA1c (Glycated Hemoglobin) $>6.5 \%$, fasting sugar $>126 \mathrm{mg} / \mathrm{dL}$ on at least 2 separate measurements, or taking anti-diabetic agents. The stroke diagnosis was according to brain imaging studies and the neurologists' documentation. All classifications were determined by an independent review of the patients' medical record. Patients with primary aldosteronism identified by confirmatory tests, imaging studies, and/or NP-59 scintigraphy consistent with recommendations of the Taiwan Society of Aldosteronism [12] were excluded, as were patients with treatable proclaimed secondary causes, stage $5 \mathrm{CKD}$ and patients on hemodialysis. We reviewed the patients' baseline characteristics, prescribed antihypertensive medications, and pre-/post-procedural changes in BP via the electronic medical records of three hospitals around Taipei.

\subsection{Predictive triad at baseline}

Patients were divided into two groups, including control patients (group A) and those meeting at least one criteria in the predictive triad (group B). This preprocedural triad consisted of the following characteristics prior to RDN: (1) more than $10 \mathrm{mmHg}$ of $\mathrm{BP}$ reduction after at least 14-day use of aldosterone antagonist, (2) ratio of serum aldosterone concentration to plasma renin activity (ARR) $>30$, or (3) the presence of slow flow on the renal angiogram. Slow flow refers to TIMI-2 flow, which is defined as delayed or sluggish antegrade flow with complete filling of the distal territory [13]. The ARR was calculated at the time of baseline 24-hour ambulatory BP measurement (ABPM) on contemporary antihypertensive medications.

\subsection{Blood pressure and antihypertensive medications}

Most patients had office BP measurement at 0, 1, 3, 6 and 12 months and 24-hour ABPM at 0, 1, 3-6 and 12 months after RDN. The medication was adjusted by the physicians' decision for clinical needs. The number of medications prescribed was recorded at $0,1,3,6$, and 12 months by reviewing the electronic medical records.

\subsection{Definition of $R D N$ responder}

Consistent with previous studies, RDN responders were defined by any of the following criteria: (1) 24-hour systolic BP reduction $\geq 6 \mathrm{mmHg}$ without increasing antihypertensive medication, or (2) withdrawal of $\geq 1$ class of antihypertensive medication $[1,2,14]$. Otherwise, the patient was labeled a non-responder.

\subsection{Statistical analyses}

All data were analyzed using SPSS version 24.0 for Windows (IBM Corp., Chicago, IL, USA). Comparison of the baseline characteristics and blood pressure during times of follow-up between group A and B were examined using the Mann-Whitney $U$ test for continuous variables, the Fisher's exact test for categorical variables and analysis of covariance (ANCOVA) for baseline difference adjustment.

\section{Results}

\subsection{Patient characteristics}

After excluding 3 cases with stage 5 CKD, 46 consecutive patients were enrolled in the study, of which 27 (59\%) were in group A and 19 (41\%) in group B. Five of 46 patients $(11 \%)$ had two features of the triad and none had all three. The regimen of aldosterone antagonist was spironolactone $25 \mathrm{mg}$ once daily. No significant differences between the groups in baseline patient demographics, BP, comorbidities, or neurohormones were observed. However, group A patients had a numerically higher mean 24-hour heart rate $(70.7 \mathrm{bpm}$ vs. $65.3 \mathrm{bpm}, p=0.071)$ and comparable 24-hour systolic (140.0 $\mathrm{mmHg}$ vs. $144.0 \mathrm{mmHg}, p=$ $0.577)$ and diastolic BP (77.5 bpm vs. $76.6 \mathrm{bpm}, p=0.928)$. Group A subjects were prescribed fewer antihypertensive agents ( 4.0 vs. $4.9, p=0.014)$ and fewer aldosterone antagonist $(3.7 \%$ vs. $47.4 \%, p=0.001)$. The proportion of CKD $(55.6 \%$ vs. $80 \%, p=0.350)$ and angiotensin-converting enzyme inhibitors/angiotensin receptor blockers (66.7\% vs. $89.5 \%, p=0.092$ ) was lower in group A, but without statistical significance (Table 1$)$. The baseline ARR was 8.5 and 93.5 in group A and B $(p=0.012)$, respectively. Aldosterone antagonist was associated with a systolic BP reduction of $11 \mathrm{mmHg}$ in those prescribed the drug. There were no procedure-related complications.

\subsection{Office and ambulatory blood pressure}

On average, office systolic BP was $154.4 \pm 22.8$ $\mathrm{mmHg}$ in group A and $164.3 \pm 27.7 \mathrm{mmHg}$ in group $\mathrm{B}(p$ 
Table 1. Baseline characteristics.

\begin{tabular}{|c|c|c|c|}
\hline Characteristic & Group A $(\mathrm{n}=27)$ & Group B $(n=19)$ & $p$ \\
\hline Age (y) & $57.4 \pm 13.4$ & $62.3 \pm 13.2$ & 0.220 \\
\hline Men & $66.7 \%$ & $52.6 \%$ & 0.373 \\
\hline \multicolumn{4}{|l|}{ Blood pressure } \\
\hline Office SBP (mmHg) & $154.4 \pm 22.8$ & $164.3 \pm 27.7$ & 0.190 \\
\hline Office DBP (mmHg) & $82.8 \pm 17.4$ & $87.4 \pm 16.9$ & 0.382 \\
\hline Office pulse pressure $(\mathrm{mmHg})$ & $71.6 \pm 16.5$ & $77.0 \pm 19.5$ & 0.316 \\
\hline Mean 24-hour SBP (mmHg) & $140.0 \pm 12.8$ & $144.0 \pm 16.5$ & 0.577 \\
\hline Mean 24-hour DBP (mmHg) & $77.5 \pm 11.2$ & $76.6 \pm 9.9$ & 0.928 \\
\hline Mean 24-hour pulse pressure (mmHg) & $62.5 \pm 12.0$ & $67.4 \pm 19.5$ & 0.386 \\
\hline Mean 24-hour HR (beat per min) & $70.7 \pm 8.7$ & $65.3 \pm 7.2$ & 0.067 \\
\hline \multicolumn{4}{|l|}{ Comorbidity } \\
\hline eGFR $\left(\mathrm{mL} / \mathrm{min} / 1.73 \mathrm{~m}^{2}\right)$ & $65.2 \pm 25.1$ & $48.7 \pm 28.5$ & 0.712 \\
\hline Chronic Kidney Disease & $55.6 \%$ & $80 \%$ & 0.350 \\
\hline Diabetes & $77.8 \%$ & $50.0 \%$ & 0.350 \\
\hline Previous stroke & $22.2 \%$ & $20.0 \%$ & 1.000 \\
\hline \multicolumn{4}{|l|}{ Medication } \\
\hline Number of medication class & $4.0 \pm 1.3$ & $4.9 \pm 0.9$ & 0.011 \\
\hline $\mathrm{ACEi}$ or $\mathrm{ARB}$ & $66.7 \%$ & $89.5 \%$ & 0.092 \\
\hline Calcium-channel blocker & $88.9 \%$ & $100.0 \%$ & 1.000 \\
\hline Beta blocker & $77.8 \%$ & $73.7 \%$ & 0.588 \\
\hline Alpha blocker & $37.0 \%$ & $57.9 \%$ & 0.231 \\
\hline Central alpha2 agonist & $14.8 \%$ & $5.3 \%$ & 0.387 \\
\hline Direct-acting vasodilator & $51.9 \%$ & $63.2 \%$ & 0.551 \\
\hline Loop diuretics or thiazide & $55.6 \%$ & $52.6 \%$ & 1.000 \\
\hline Aldosterone antagonist & $3.7 \%$ & $47.4 \%$ & 0.001 \\
\hline \multicolumn{4}{|l|}{ Neurohormone } \\
\hline Aldosterone (ng/dL) & $51.5 \pm 52.2$ & $61.0 \pm 68.1$ & 0.654 \\
\hline Renin activity (ng/mL/hr) & $16.6 \pm 25.0$ & $7.0 \pm 11.1$ & 0.160 \\
\hline \multicolumn{4}{|c|}{$\begin{array}{l}\text { ACEi, angiotensin-converting enzyme inhibitor; ARB, angiotensin receptor blockers; DBP, di- } \\
\text { astolic blood pressure; eGFR, estimated glomerular filtration rate; HR, heart rate; SBP, systolic } \\
\text { blood pressure. }\end{array}$} \\
\hline
\end{tabular}

$=0.310$ ) at baseline. At 3- and 6-months post RDN, mean office systolic BP was $137.9 \mathrm{mmHg}$ vs. $133.2 \mathrm{mmHg}$, respectively. Among the groups, the average reduction in office systolic BP was similar $(-18.5 \pm 22.8 \mathrm{mmHg}$ vs. $-19.3 \pm 27.6 \mathrm{mmHg}, p=0.916)$ at 1 month. However, the difference between group $\mathrm{A}$ and $\mathrm{B}$ became greater at 3 months $(-14.3 \pm 23.2 \mathrm{mmHg}$ vs. $-30.7 \pm 29.8 \mathrm{mmHg}$, $p=0.057)$ and 12 months $(-12.4 \pm 23.5 \mathrm{mmHg}$ vs. -29.9 $\pm 25.5 \mathrm{mmHg}, p=0.046$ ) (Table 2). The difference at 12 months remained significant after adjusting the baseline BP ( $p$ for model $1=0.048$ ) and other confounding factors ( $p$ for model $2=0.025$ ) (Table 2). Observed differences at 6and 12-months post RDN might have been attenuated due to drug withdraw, especially in the group B. Mean 24-hour systolic BP at baseline was $140.0 \pm 12.8 \mathrm{mmHg}$ vs. 144.0 $\pm 16.5 \mathrm{mmHg}(p=0.577)$. At 1 -month post $\mathrm{RDN}$, the 24 - hour systolic BP decreased from $132.5 \pm 17.7 \mathrm{mmHg}$ to $136.3 \pm 14.0 \mathrm{mmHg}$. Numerically, the decline in 24-hour systolic BP was greater in group B at 3-6 months $(-9.4 \pm$ $19.6 \mathrm{mmHg}$ vs. $-18.3 \pm 12.3, p=0.330)$ and 12 months $(-$ $8.9 \pm 17.0 \mathrm{mmHg}$ vs. $-12.5 \pm 5.3, p=0.693)$, respectively (Table 2).

\subsection{Response to renal denervation}

The percentage of drug classes in use by each group is depicted in Fig. 1; the proportion of patients in group B prescribed at least four classes of drug decreased from $95 \%$ to $73 \%$ within three months and to $62 \%$ at 12 months. Compared to group A, the number of drug classes continued to decrease to 12 months in group B. 
Table 2. Office and 24-hour systolic blood pressure at times of follow-up.

\begin{tabular}{|c|c|c|c|c|c|c|c|c|c|}
\hline & \multicolumn{5}{|c|}{ Office SBP (mmHg) } & \multicolumn{4}{|c|}{ 24-hour SBP (mmHg) } \\
\hline & Baseline & 1 month & 3 months & 6 months & 12 months & Baseline & 1 month & $3-6$ months & 12 months \\
\hline $\begin{array}{l}\text { Patient number } \\
\text { Group A vs. B }\end{array}$ & 27 vs. 19 & 25 vs. 17 & 22 vs. 18 & 20 vs. 15 & 19 vs. 15 & 19 vs. 14 & 13 vs. 11 & 12 vs. 9 & 7 vs. 4 \\
\hline Group A & $154.4 \pm 22.8$ & $137.4 \pm 17.0$ & $137.9 \pm 15.6$ & $137.9 \pm 17.9$ & $144.9 \pm 18.9$ & $140.0 \pm 12.8$ & $132.5 \pm 17.7$ & $129.9 \pm 11.5$ & $133.4 \pm 12.6$ \\
\hline \multirow[t]{3}{*}{ Group B } & $164.3 \pm 27.7$ & $142.7 \pm 19.9$ & $133.2 \pm 23.3$ & $133.2 \pm 20.6$ & $131.0 \pm 19.0$ & $144.0 \pm 16.5$ & $136.3 \pm 14.0$ & $134.2 \pm 16.3$ & $131.2 \pm 4.1$ \\
\hline & \multicolumn{5}{|c|}{$\Delta$ Office SBP $(\mathrm{mmHg})$} & \multicolumn{4}{|c|}{$\Delta$ 24-hour SBP (mmHg) } \\
\hline & & 1 month & 3 months & 6 months & 12 months & & 1 month & $3-6$ months & 12 months \\
\hline Group A & & $-18.5 \pm 22.8$ & $-14.3 \pm 23.2$ & $-13.4 \pm 27.2$ & $-12.4 \pm 23.5$ & & $-7.7 \pm 16.0$ & $-9.4 \pm 19.6$ & $-8.9 \pm 17.0$ \\
\hline Group B & & $-19.3 \pm 27.6$ & $-30.7 \pm 29.8$ & $-26.9 \pm 34.5$ & $-29.9 \pm 25.5$ & & $-7.3 \pm 12.9$ & $-18.3 \pm 12.3$ & $-12.5 \pm 5.3$ \\
\hline$p$ & & 0.916 & 0.057 & 0.748 & 0.046 & & 0.398 & 0.330 & 0.693 \\
\hline$p$ for model 1 & & 0.465 & 0.256 & 0.495 & 0.048 & & 0.373 & 0.961 & 0.707 \\
\hline$p$ for model 2 & & 0.421 & 0.052 & 0.459 & 0.025 & & 0.900 & 0.629 & 0.430 \\
\hline
\end{tabular}

SBP, systolic blood pressure. Group A: triad (-); Group B: triad (+).

$\Delta$, change in blood pressure after renal denervation.

$p$ for model 1: adjusting for baseline SBP, age and gender by analysis of covariance (ANCOVA).

$p$ for model 2: adjusting for baseline office SBP, DBP, age, gender, number of medication class, aldosterone, and renin activity by analysis of covariance (ANCOVA).

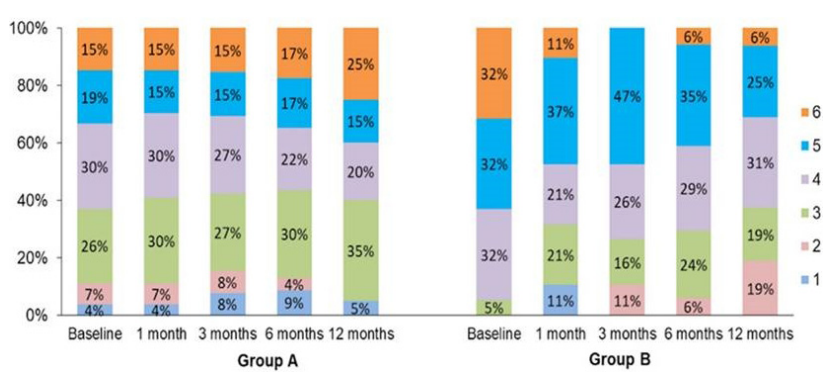

Fig. 1. Percentage of drug class number distribution. Group A: triad (-); Group B: triad (+). The percentage of drug classes in use is depicted at baseline and 1, 3, 6, 12 months. The percentage of patients taking less than four classes of drug increases till 12 months only in group B.

Overall, nineteen patients ( $41 \%$ ) had response to RDN in the first month. Thirty (65\%) and thirty-one $(67 \%)$ of all patients responded to the RDN procedure in six months and twelve months, respectively. The response rate was significantly greater in group B at 3 months $(48.1 \%$ vs. $84.2 \%$, $p=0.016)$; and $(48.1 \%$ vs. $89.5 \%, p=0.005)$ at 6 months. The proportion and timeline of response in two groups are depicted in Fig. 2.

\section{Discussion}

Sympathetic nerveous system activity is associated with RAAS overdrive in neurogenic hypertension [15]. The RAAS may have a critical role in the activation of the sympathetic nervous system. Accumulated basic and clinical evidence supports the use of inhibitors of the RAAS, including aldosterone antagonists. These findings are con-

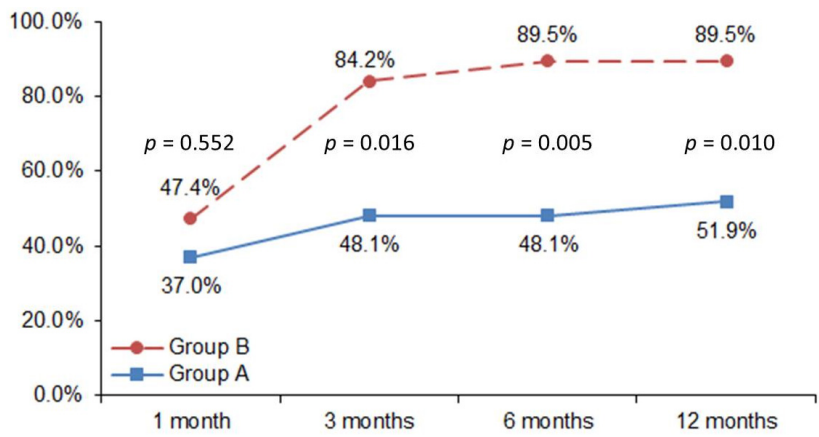

Fig. 2. Response rate to renal denervation (RDN) by groups. Group A: triad (-); Group B: triad (+). The change in proportion of responders in both groups during 12 months after RDN.

sistent with the result that RDN significantly reduces the level of renin and aldosterone [5]. This study demonstrated that RDN was associated with a greater response in group B patients, who had predictive features suggesting occult aldosterone overactivity. RDN may further inhibit preexisting sympathetic activity in patients with relatively active RAAS, which was inadequately suppressed by concomitant antihypertensive medications.

Due to its complexity, the concept of aldosteronism remains incompletely understood, and its true prevalence remains uncertain. Usually, the differential diagnosis would be initiated when ARR $>30$, suggestive of the presence of aldosteronism [16,17]. Compared to the well-established criteria for primary aldosteronism, the secondary type depends on a diagnosis of exclusion [12,18]. Several studies have reported that higher baseline heart rate and plasma renin activity predict better RDN response in drug-naive pa- 
tients [5,19]. However, its influence in patients on treatment remains unclear. The on-treatment ARR in this study helps identify residual aldosterone overactivity. Also, patients with occult RAAS overactivity could be present when having greater BP response to aldosterone inhibition than expected, i.e., more than $10 \mathrm{mmHg}$. The present results indicate that a patients' prior response to an aldosterone antagonist might be an important predictor of RDN response.

Impaired renal blood flow secondary to systemic disorders is a common etiology of RAAS overactivity [10,20]. Therefore, decreased renal blood flow (TIMI-2) on the renal angiogram was another feature we chose to identify patients with overactive RAAS. Notably, the effect of BP lowering by angioplasty alone is disappointing [21]. However, RDN has been shown to increase renal blood flow in animals [22], and investigating its impact on BP control is worthy of prospective investigation.

The patients in group B showed comparable BP effects at 1 month and more delayed response from 3 to 6 months. These findings might suggest a possible mechanism for to explain continuous BP decline for months in randomized control trials [1,23]. Decreased spillover of norepinephrine could happen soon after RDN, but the process to modulate RAAS could last much longer. Notably, the SPYRAL HTN-ON MED trial also showed an apparent time effect of gradually decreasing systolic BP in the RDN treated group compared to the sham control group [1]. However, the present 24-hour BP measurements at 1 month also seem to indicate that RDN could help to lower BP immediately within one month in nearly half of all RDN recipients.

This multi-center, retrospective post-hoc analysis has several limitations, including the relatively small sample size and imbalanced medication choices. The imbalanced RAAS inhibitors could also reflect the discriminative power of predictive triad. BP change may be attenuated by medication adjustment in the late period of follow-up; however, we still observed significant reduction of office BP at 12 months in group B compared with group A. Because our study cohort was not drug-naïve, the determinant ARR could be influenced by multiple drug interactions, leading to nonspecific diagnosis in both groups. So, the predictive triad should not be interpreted as criteria of diagnosis. It is possible that a small proportion of patients in group A may have unknowingly met one of the triad criteria due to missing data. However, the difference in proportion still led to a lower response rate in group A.

\section{Conclusions}

Patient selection under medications remains an important unmet clinical need. We observed a superior response rate following RDN in the group with the predictive triad at baseline. Our results warrant further investigation on its application and causality.

\section{Author contributions}

SIL and YHL contributed to the conception and design of the study. SIL, SHS, LYML, PLL, WRL, CLC, WRC, CTT, YJW, TDW, YHL collected the data. $\mathrm{CCH}$ and YHL performed and supervised statistical analysis. SIL drafted the manuscript. SIL, CCH, YHL critically reviewed the manuscript. All authors read and approved the final manuscript.

\section{Ethics approval and consent to participate}

This study was approved by the Institutional Review Boards of MacKay Memorial Hospital (approval number: 20MMHIS087e), which waived the requirement for informed consent in this retrospective study.

\section{Acknowledgment}

We would like to express our gratitude to all those who have supported our work. Thanks to all the peer-reviewers for their opinions and suggestions.

\section{Funding}

This research received no external funding.

\section{Conflict of interest}

The authors declare no conflict of interest. Tzung-Dau Wang is serving as one of the Editorial Board members of this journal. We declare that Tzung-Dau Wang had no involvement in the peer review of this article and has no access to information regarding its peer review. Full responsibility for the editorial process for this article was delegated to Stefano Omboni, Brian Tomlinson and Takatoshi Kasai.

\section{References}

[1] Kandzari DE, Bohm M, Mahfoud F, Townsend RR, Weber MA, Pocock S, et al. Effect of renal denervation on blood pressure in the presence of antihypertensive drugs: 6-month efficacy and safety results from the SPYRAL HTN-ON MED proof-ofconcept randomised trial. Lancet. 2018; 391: 2346-2355.

[2] Bohm M, Kario K, Kandzari DE, Mahfoud F, Weber MA, Schmieder RE, et al. Efficacy of catheter-based renal denervation in the absence of antihypertensive medications (SPYRAL HTN-OFF MED Pivotal): a multicentre, randomised, shamcontrolled trial. Lancet. 2020; 395: 1444-1451.

[3] Azizi M, Schmieder RE, Mahfoud F, Weber MA, Daemen J, Davies J, et al. Endovascular ultrasound renal denervation to treat hypertension (RADIANCE-HTN SOLO): a multicentre, international, single-blind, randomised, sham-controlled trial. Lancet. 2018; 391: 2335-2345.

[4] Azizi M, Schmieder RE, Mahfoud F, Weber MA, Daemen J, Lobo MD, et al. Six-Month Results of Treatment-Blinded Medication Titration for Hypertension Control Following Randomization to Endovascular Ultrasound Renal Denervation or a Sham Procedure in the RADIANCE-HTN SOLO Trial. Circulation. 2019; 139: 2542-2553.

[5] Mahfoud F, Townsend RR, Kandzari DE, Kario K, Schmieder RE, Tsioufis K, et al. Changes in Plasma Renin Activity after Renal Artery Sympathetic Denervation. Journal of the American College of Cardiology. 2021; 77: 2909-2919. 
[6] Fisher NDL, Kirtane AJ, Daemen J, Rader F, Lobo MD, Saxena $\mathrm{M}$, et al. Plasma renin and aldosterone concentrations related to endovascular ultrasound renal denervation in the RADIANCEHTN SOLO trial. Journal of Hypertension. 2022; 40: 221-228.

[7] Böhm M, Tsioufis K, Kandzari DE, Kario K, Weber MA, Schmieder RE, et al. Effect of Heart Rate on the Outcome of Renal Denervation in Patients with Uncontrolled Hypertension. Journal of the American College of Cardiology. 2021; 78: 10281038.

[8] Bhatt DL, Kandzari DE, O'Neill WW, D'Agostino R, Flack JM, Katzen BT, et al. A controlled trial of renal denervation for resistant hypertension. The New England Journal of Medicine. 2014; 370: 1393-1401.

[9] Kandzari DE, Bhatt DL, Brar S, Devireddy CM, Esler M, Fahy $\mathrm{M}$, et al. Predictors of blood pressure response in the SYMPLICITY HTN-3 trial. European Heart Journal. 2015; 36: 219-227.

[10] Perazella MA, Setaro JF. Renin-angiotensin-aldosterone system: fundamental aspects and clinical implications in renal and cardiovascular disorders. Journal of Nuclear Cardiology. 2003; 10: 184-196.

[11] Lin SI, Lin PL, Huang CC, Liu LYM, Chiou WR, Lan WR, et al. Renal Denervation May Benefit Aldosteronism-Related Hypertension-A Single-Center Experience. Strait Circulation Journal. 2020; 2: 13-20.

[12] Wu V, Hu Y, Er LK, Yen R, Chang C, Chang Y, et al. Case detection and diagnosis of primary aldosteronism - the consensus of Taiwan Society of Aldosteronism. Journal of the Formosan Medical Association. 2017; 116: 993-1005.

[13] TIMI Study Group. The Thrombolysis in Myocardial Infarction (TIMI) Trial. Phase I findings. New England Journal of Medicine. 1985; 312: 932-936.

[14] Liu LY, Lin P, Liao F, Lin S, Chiou W, Wu Y, et al. Effect of Radiofrequency-Based Renal Denervation: the Impact of Unplanned Medication Change from a Systematic Review and Meta-Analysis. Acta Cardiologica Sinica. 2019; 35: 144-152.

[15] Biancardi VC, Sharma NM. Connecting sympathetic and renin-angiotensin system overdrive in neurogenic hypertension through miRNA-181a. Hypertension Research. 2020; 43: 13091310.

[16] Calhoun DA, Jones D, Textor S, Goff DC, Murphy TP, Toto RD, et al. Resistant hypertension: diagnosis, evaluation, and treatment: a scientific statement from the American Heart Association Professional Education Committee of the Council for High Blood Pressure Research. Circulation. 2008; 117: e510-e526.

[17] Wang T, Lee Y, Chang S, Tung Y, Yeh C, Lin Y, et al. 2019 Consensus Statement of the Taiwan Hypertension Society and the Taiwan Society of Cardiology on Renal Denervation for the Management of Arterial Hypertension. Acta Cardiologica Sinica. 2019; 35: 199-230.

[18] Calhoun DA. Aldosteronism and hypertension. Clinical Journal of the American Society of Nephrology. 2006; 1: 1039-1045.

[19] Bohm M, Mahfoud F, Townsend RR, Kandzari DE, Pocock S, Ukena $\mathrm{C}$, et al. Ambulatory heart rate reduction after catheterbased renal denervation in hypertensive patients not receiving anti-hypertensive medications: data from SPYRAL HTN-OFF MED, a randomized, sham-controlled, proof-of-concept trial. European Heart Journal. 2019; 40: 743-751.

[20] Carlström M, Wilcox CS, Arendshorst WJ. Renal autoregulation in health and disease. Physiological Reviews. 2015; 95: 405511.

[21] Cooper CJ, Murphy TP, Cutlip DE, Jamerson K, Henrich W, Reid DM, et al. Stenting and medical therapy for atherosclerotic renal-artery stenosis. The New England Journal of Medicine. 2014; 370: 13-22.

[22] Pellegrino PR, Zucker IH, Chatzizisis YS, Wang H, Schiller AM. Quantification of Renal Sympathetic Vasomotion as a Novel End Point for Renal Denervation. Hypertension. 2020; 76: $1247-$ 1255.

[23] Mahfoud F, Mancia G, Schmieder R, Narkiewicz K, Ruilope L, Schlaich M, et al. Renal Denervation in High-Risk Patients with Hypertension. Journal of the American College of Cardiology. 2020; 75: 2879-2888. 